\title{
Efficacy of a polyvalent mastitis vaccine against Staphylococcus aureus on a dairy Mediterranean buffalo farm: results of two clinical field trials
}

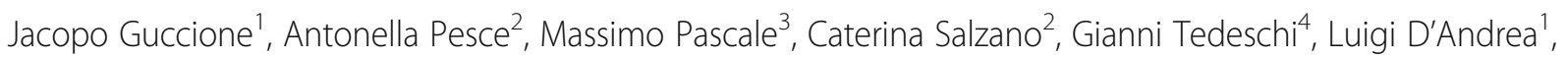
Angela De Rosa ${ }^{1}$ and Paolo Ciaramella ${ }^{1 *}$

\begin{abstract}
Background: In the last years the knowledges on Mediterranean Buffalo (MB) mastitis is remarkably improving, nevertheless the attention has been never focused on vaccination as preventive strategy for the control of mastitis in these ruminates. The aim of the current study was to assess clinical efficacy over time of two different preventive vaccination protocols against S. aureus mastitis, in primiparous MB.Vaccinated (VG) and not-vaccinated (N-VG) groups, of $30 \mathrm{MB}$ each one, were selected from two different herds (herd A: VG1 and N-VG1; herd B: VG2 and NVG2) of the same farm. Herd A received a double vaccination (Startvac ${ }^{\oplus}, 45$ and 10 days before calving, protocol A), while in herd B an additional administration was performed (52 days after calving, protocol B). Bacteriological milk culture and assessment of somatic cell count (SCC) were performed at 10, 30, 60 and 90 days in milk (DIM) from composite milk samples. After 90 DIM, daily milk yields and SCC values were monthly detected until dry-off.
\end{abstract}

Results: The overall incidence of positive MB for S. aureus was 40.8\% (49/120) in VG1 and 43.3\% (52/120) in N-VG1 (Protocol A), while 45.8\% (55/120) and 50.8\% (61/120) in VG2 and N-VG2 (Protocol B). The latter was associated with a significant decreased in prevalence (at $90 \mathrm{DIM}$ ) and incidence of mastitis (animals positive for S. aureus, SCC $>200 \wedge 10^{3}$, with or without clinical signs) in the vaccinated MB, while no difference occurred in protocol A. Moreover, herd B showed a significant reduction in prevalence of intramammary infection (animals positive for $S$. aureus, SCC $<200 \wedge 10^{3}$, no clinical signs) in the vaccinated MB at 60 DIM while no differences were detected in herd A, at any sampling time; N-VG2 had significantly higher overall SCC values than VG2 (4.97 \pm 4.75 and $4.84 \pm 4.60$ Log10 cells $/ \mathrm{mL} \pm$ standard deviation, respectively), while no differences were recorded in herd A.

Conclusions: The current investigation explores for the first time the clinical efficacy of vaccinations against $S$. aureus infections in $\mathrm{MB}$, showing encouraging results regarding reduction in mastitis and somatic cell count; the polyvalent mastitis vaccine may be considered an additional tool for in-herd $S$ aureus infection and should be associated to other control procedures to maximize its properties.

Keywords: Staphylococcus aureus, Vaccination, Prophylaxis, Bubalus bubalis, Udder health

\footnotetext{
* Correspondence: paociara@unina.it

1 Department of Veterinary Medicine and Animal Productions, University of

Napoli "Federico II", Via Delpino 1, 80137 Naples, Italy

Full list of author information is available at the end of the article
} 


\section{Background}

Mastitis represents one of the major causes of economic loss in dairy Mediterranean buffaloes (MB) because negatively influencing milk quality and yields of the animals affected [1, 2]. In MB mastitis is mainly caused by several contagious (e.g. Staphylococcus aureus, Streptococcus agalactiae), environmental (e.g. Streptococcus uberis and Streptococcus dysgalactiae, Escherichia coli, Enterobacteriaceae, and yeasts), and opportunistic (e.g. coagulase-negative staphylococci) bacteria [3-5].

$S$. aureus is one of the most important pathogens, causing clinical and subclinical mastitis in dairy $\mathrm{MB}$ and cows all over the world [2, 6, 7]; Clinical outcomes and high within-herd prevalence were recently described in MB confirming its relevance as contagious microorganism [2]. This bacteria typically colonizes the injured skin; damage of the teat end and faulty milking encourages migration of bacteria into the udder causing intramammary infections (IMI) and sometimes persisting for extended periods $[4,8,9]$. Some strains are particularly resistant to antibiotics [4] and moderate results were reported in an our previous study exploring the effects of an antibiotic selective treatment [1]. As a consequence, similarly to dairy cows additional strategies of herd health management including treatment or culling of affected animals, implementation of biosecurity measures and hygienic milking practices, have been recommended to prevent and control udder health problems related to S. aureus in MB farms [2].

Although with different outcomes, great scientific attention was recently given to mastitis control by means of preventive vaccination protocols in cows [10-16]. A commercial multivalent vaccine containing inactivated $S$. aureus and E. coli has been available in European Union for the last years and several investigations regarding its usefulness were recently performed in cows with different results $[13,14,17]$. In $\mathrm{MB}$, the awareness for mastitis are remarkably improving, although the attention has been never focused before on vaccination as preventive strategy for mastitis.

Considering the premises, the aims of the present study was to evaluate the clinical efficacy of a polyvalent commercial vaccine administered in dairy primiparous $\mathrm{MB}$ evaluating as outcomes (1) the prevalence and incidence of IMI and mastitis, (2) the effects on somatic cell count and (3) milk yield.

\section{Methods}

\section{Animals and Farm Management}

All the animals chosen in the present study were reared in the same breeding farm of approximately 700 dairy buffaloes, free from mandatory reportable diseases and located in Caserta area (Southern Italy). The eligible criteria for the farm were represented by 3 , monthly and consecutive samplings of bulk tank milk positive for $S$. aureus before the beginning of the study, analyzed by means of PCR-based assay as described by Syring et al. [18]. No strict criteria were instead applied for bulk milk somatic cell count (SCC) or mastitis incidence. Differences observed about herd management practices were recorded during the period of the investigation (two consecutive years) to exclude possible influences on vaccination efficacy.

Farm was characterized by herringbone parlor and animals were milked twice a day. A mean of $247 \pm 23$-day milk yield per head of $2200 \mathrm{~kg}$ while mean bulk tank SCC values of $172 \times 10^{3} \pm 49 \times 10^{3}$ and $179 \times 10^{3} \pm 32 \times$ $10^{3}$ cell $/ \mathrm{mL}$ were recorded for the whole herd during the first and the second year of investigation, respectively. All MB selected were kept in a roofed common paddock of $\sim 1200 \mathrm{~m}^{2}(\sim 30 \mathrm{~m} \times 40 \mathrm{~m})$ As often observed in MB's farms, before parturition and until 40-50 DIM, all the primiparous $\mathrm{MB}$ were housed separated from the pluriparous. Milking was performed in the same milking parlor, although the primiparous animals were milked first. After 50 DIM, primiparous were mixed with the pluriparous animals, including milking time. The barn was characterized by a common bedded area (with dried manure solids), a loafing area and a feeding alley with solid non-grooved concrete floor (cleaned twice a day). The entire herd was fed a total mixed ratio including hay, silage and a multi-vitamin integrator three times a day; free access to the protected water trough was always guaranteed.

\section{Study design}

Two herds (A and B) were enrolled in the current study. Sixty primiparous for each herd were subdivided into two groups of $30 \mathrm{MB}$ : vaccinated and not-vaccinated (herd A: VG1 and N-VG1; herd B: VG2 and N-VG2). Animals were chosen from the same farm in two consecutive lactations (herd A: I year, December 2012 to November 2013 - herd B: II year, December 2013 to November 2014). Every year the animals were chosen by means convenience sampling from 80 primiparous in good health having 4 functional quarters, teats free of significant lesions, and with an estimated calving date to allow vaccination at predicted times before calving. Samples size was calculated by using the formula proposed by Thrusfield [19] considering the following values: study population (pregnant primiparous $\mathrm{MB}$ in farm, $\sim 14 \%$ of entire herd - approximately stable parameter in both years considered), expected prevalence of positive primiparous $\mathrm{MB}$ for $S$. aureus (55\%) [1], confidence interval $95 \%$ and desired absolute precision (5\%).

All the animals were individually submitted to a complete clinical examination with particular focus on 
the udder health status following the clinical procedure described by Ciaramella [20].

For each herd the progressive vaccination was performed on $50 \mathrm{MB}$ were chosen by means of simple randomization; out of the latter, the first 30 animals reaching the entire vaccination protocol were enrolled as VG. The other 30 not vaccinated subjects were selected as N-VG.

On sampling day, local and systemic signs and changes in milk appearance, such us overall appearance of depression, udder swelling and pain, off-color and watery appearance of milk, and presence of flakes, clots or pus, were recorded. California Mastitis Test (CMT) was routinely performed from each composite milk sample, with values $\geq 1$ interpreted as positive [2] (data not shown).

\section{Vaccine}

Commercial polyvalent mastitis vaccine (STARTVAC ${ }^{\ominus}$, HIPRA Spain Co. Ltd, Girona) containing inactivated $E$. coli 55 and inactivated $S$. aureus (CP8) strains SP 140, expressing slime-associated antigenic complex (SAAC) was used [21].

The farmer was previously informed about the purposes and methods of the two clinical field-trials and the study received the approval by the Ethical Animal Care and Use Committee of University of Studies of Naples "Federico II". During the present investigation each herd was submitted to a different vaccination protocol: the first (protocol A - herd A) was based on two intramuscular vaccine injections located at the medium third of the neck ( $2 \mathrm{~mL}$ each one) and performed at 45 and 10 days before the estimated date of calving on VG1; the second one (protocol B - herd B), instead included an additional administration on VG2 at 52 days in milk (DIM).

\section{Milk Samples}

All milk samples were aseptically collected in sterile test tubes immediately before regular evening milking, as described by National Mastitis Council [22] for dairy cows. At 10, 30, 60 and 90 DIM, a sample from each quarter was collected; a composite milk sample was created by mixing equal amounts of milk $(50 \mathrm{~mL})$ from all 4 quarters into a sterile test tube (BD Vacutainer, Oxford, UK) and used to perform bacteriological milk culture (BMC) and determine the SCC; finally, milk yields were also recorded during the same samplings time using automatic dedicated software (Afifarm, Afimilk, Kibbutz Afikim, Israel). After 90 DIM, milk yield and SCC values were evaluated at 150 and 240 DIM (close to dry off). As described by a previous study [1], a great variability to achieve the supposed drying-off time (daily milk yield < $0.5 \mathrm{~L} /$ day) was detected in the enrolled animals (from 240 to 260 DIM), as consequence, the last sample considered useful for the statistical and economic analysis was collected at 240 DIM.

\section{Definition of Udder Health Status}

Following the classification reported in previous studies $[1,23]$, the animals enrolled were defined as negative for S. aureus, affected by IMI or mastitis considering: presence/absence of clinical signs, SCC values, and microbiological status; briefly, MB producing milk with SCC below the threshold of $200 \times 10^{3}$ cells $/ \mathrm{mL}$ and associated with BMC negative to $S$. aureus were considered and hereafter referred to as "healthy udder"; animals with analogous SCC values and positive BMC caused by $S$. aureus have been instead considered affected by IMI; moreover, buffaloes producing milk with SCC upon the threshold of $200 \times 10^{3}$ and positive BMC for S. aureus have been defined as affected by mastitis, with (clinical mastitis - CM) or without clinical signs (subclinical mastitis - SCM) [1, 23]. All the MB affected by mastitis (CM and/or SCM) due to $S$. aureus were considered in endpoint phase and excluded from the study.

\section{Cytological and bacteriological investigations}

Composite milk samples were placed in a cool box $\left(4{ }^{\circ} \mathrm{C}\right)$ and brought to the reference laboratory within $1 \mathrm{~h}(\mathrm{~h})$ of collection, where they were submitted to SCC analysis and BMC within $2 \mathrm{~h}$ of collection. SCC was determined using an automatic analyzer, approved for buffalo milk (Fossomatic 5000, Foss Electric, Hillerod, Denmark) [24]. The BMC and bacteriological identification were performed according to guidelines of the National Mastitis Council [25]. Briefly, $10 \mu \mathrm{L}$ of each milk sample was streaked on one quarter of a blood-agar plate (Merck KGaA, Darmstadt, Germany), incubated at $37{ }^{\circ} \mathrm{C}$ for up to $48 \mathrm{~h}$ and examined two time (at 24 and $48 \mathrm{~h}$ of incubation). Bacterial colonies were tentatively identified on gross morphology; number and types of colonies were also recorded. As described in previous studies [2], when 3 or more dissimilar colony types were isolated on the plate, the sample was considered contaminated. Gram staining and catalase testing were performed to differentiate between streptococci and staphylococci, and tube coagulase testing using rabbit plasma to differentiate between coagulase-positive and coagulase-negative staphylococci. A final identification of microorganisms was performed using the colorimetric automated identification system (Vitek 2 XL 120; bioMerieux Inc., Hazelwood, MO), according to the manufacturer's instructions. Enterobacteriaceae were grown on MacConkey agar (Oxoid, Basingstoke, UK) and identified using the same automated system. Isolates identified with confidence levels greater than 0.90 were considered identified mastitis pathogens at species level; otherwise, they were identified at genus level.). All the laboratory 
procedures were performed without previous informations about the results of the clinical examination of the udder (physical and CMT).

\section{Statistical analysis}

Different animal health status, SCC, and milk yields were analyzed by standard descriptive statistics and normality was assessed using histograms, normal probability plots and Shapiro Wilk tests. Data were expressed as absolute numbers, percentage, or mean \pm SD. Log- transformation was applied to variable not normally distributed. Untransformed and log-transformed continuous variables (SCC and milk yields) were compared using Student's $t$-test. Significant differences between expected and observed frequencies of categorical data (i.e. vaccine efficacy: VG1 vs. N-VG1 and VG2 vs. N-VG2) were assessed by means of contingency tables, using $X^{2}$-test. Fisher's exact test was performed in case of low expected frequencies $(<5)$. Probabilities $<0.05$ were considered significant. Moreover, a mixed models were also used to investigate the direct effects of the vaccination intra- and inter-protocols. The final model included fixed time effect, fixed group effect, fixed interaction time*group and buffalo as random effect. For statistical analysis of the outcomes a mixed linear models was used for continuous data (SCC and milk yield); Bonferroni correction was used to set $P$-value for multiple statistical test; the threshold for statistical significance in our analysis was $P=0.0052$, the null hypothesis was rejected for $P<0.01$.

For categorical data (presence/absence of mastitis) a mixed logistic models based only on fixed group effects and buffalo-specific random effect was performed because otherwise the function to be maximized was not concave due to the small amount of observations. Data inter-protocols (absolute values) were combined in the model including time and group as fixed effects. Probabilities $<0.05$ were considered significant. All the statistical data were analyzed using dedicated software (STATA 12.0, StataCorp LP, Collage Station, Texas, USA).

\section{Results}

\section{Course of the study and prevalence of udder pathogens}

No general or local adverse reactions to the use of the vaccine was observed in all the buffaloes of both the protocols after the administrations. The request number of vaccinated animals was reached in 1.5 months in herd $\mathrm{A}$ and in 2 months in herd B. Vaccinations were performed $45.8 \pm 4.1$ and $11.1 \pm 2.4$ days pre-calving in VG1, while $46.2 \pm 4.6$ and $10.3 \pm 2.2$ days-pre calving in VG2; the additional vaccination expected for protocol B was instead administered $52.2 \pm 1.1$ days post-calving. During the two years of study, no differences about all the aspects of herd management practices (e.g. feeding and housing system, milking routine, dairy workers, therapeutic protocols for mastitis, etc.) were detected.

Throughout the study, 480 composite milk samples were collected and submitted to BMC. All udder pathogens isolated during the first 90 DIM in vaccinated and not-vaccinated groups of the two herds and their relative incidence are reported in Table 1 . Briefly, the most commonly isolated pathogens in both the groups of the two herds, were $S$. aureus followed by coagulase-negative staphylococci. Positive-culture status for $S$. aureus was detected in $30.2 \%(49 / 162)$ and $32.5 \%(52 / 160)$ of the overall bacteriological results in VG1 and N-VG1, respectively; it was instead recorded in $41.9 \%(55 / 131)$ and $50.4 \%(61 / 121)$ of the samples in VG2 and N-VG2, respectively. E. coli was only detected in both groups of herd B (VG2: 1 time, 0.8\% and N-VG2: 2 times, 1.7\%, Table 1) and associated with not severe CM (mild milk change, CMT $\geq 2$ ).

The overall percentage of positive samples for other udder pathogens, except for S. aureus and E. coli, was 29.6\% (48/162) in VG1 and 30.6\% (49/160) in N-VG1, while it was $35.9 \%(47 / 131)$ and $23.9 \%(29 / 121)$ in VG2 and N-VG2, respectively.

Negative-culture status was observed in 40.1\% (65/ $162)$ and $36.9 \%(59 / 160)$ of the all bacteriological results observed in VG1 and N-VG1, respectively; $21.4 \%$ (28/ $131)$ and $24.0 \%(29 / 121)$ were instead recorded in VG2 and N-VG2, respectively.

\section{Vaccine effects \\ Protocol A}

The $40.8 \%(49 / 120)$ and $43.3 \%(52 / 120)$ of the samples collected were positive for $S$. aureus in VG1 and N-VG1. No bacteria were detected at 10 DIM $(0 / 30)$ in VG1, while their presence increased up to $16.7 \%$ (5/30), $60.0 \%$ $(18 / 30)$ and $89.2 \%(25 / 28)$ at 30, 60 and 90 DIM, respectively; in N-VG1 the prevalence of positive-cultures for $S$. aureus was instead of 3.3\% (1/30), 33.3\% (10/30), $53.6 \%(15 / 28)$ and $92.8 \%(26 / 28)$ at $10,30,60$ and 90 DIM, respectively; no statistically significant difference was found regarding this variable between VG1 and NVG1 at any sampling time.

Details regarding of the effects of $S$. aureus on udder health are reported in Table 2. Briefly, two CM due to $S$. aureus were recorded both in VG1 $(6.6 \%, 2 / 30)$ and NVG1 $(6.6 \%, 2 / 30)$, at 60 and 30 DIM, respectively. During the investigation, no SCM due to $S$. aureus were detected between 10 and 60 DIM in both groups, while they were observed both in VG1 $(14.3 \%, 4 / 28)$ and NVG1 $(10.7 \%, 3 / 28)$ at 90 DIM. No statistically significant difference between prevalence of mastitis in VG1 and NVG1 was observed. During the first 90 DIM, no statistically significant difference was recorded between the incidence of mastitic animals in VG1 $(20.0 \%, 6 / 30)$ and 
Table 1 Bacteriological results of all samples collected in both the herds of primiparous Mediterranean buffalo during the first 90 days in milk of investigation. Percentages represent the proportion calculate on the total number bacteriological results

\begin{tabular}{|c|c|c|c|c|c|c|c|c|}
\hline \multirow[b]{3}{*}{ Pathogens } & \multicolumn{4}{|c|}{ Herd A } & \multicolumn{4}{|c|}{ Herd B } \\
\hline & \multicolumn{2}{|l|}{ VG1 } & \multicolumn{2}{|c|}{ N-VG1 } & \multicolumn{2}{|l|}{ VG2 } & \multicolumn{2}{|c|}{ N-VG2 } \\
\hline & No. & Percent & No. & Percent & No. & Percent & No. & Percent \\
\hline Staphylococcus aureus & 49 & 30.2 & 52 & 32.5 & 55 & 41.9 & 61 & 50.4 \\
\hline Coagulase-negative staphylococci & 32 & 19.9 & 32 & 19.3 & 41 & 31.3 & 16 & 13.1 \\
\hline Enterococcus faecalis & 2 & 1.2 & 2 & 1.3 & 1 & 0.8 & 1 & 0.8 \\
\hline Aerococcus viridans & 4 & 2.5 & 4 & 2.5 & 2 & 1.5 & 4 & 3.3 \\
\hline Streptococcus dysgalactiae & 2 & 1.2 & 1 & 0.6 & 0 & 0 & 2 & 1.7 \\
\hline Streptococcus thoraltensis & 2 & 1.2 & 2 & 1.3 & 0 & 0 & 1 & 0.8 \\
\hline Other gram-positive pathogens (not S. aureus) & 2 & 1.2 & 5 & 3.1 & 3 & 2.3 & 2 & 1.7 \\
\hline Escherichia coli & 0 & 0 & 0 & 0 & 1 & 0.8 & 2 & 1.7 \\
\hline Acinetobacter Iwoffii & 4 & 2.5 & 3 & 1.9 & 0 & 0 & 2 & 1.7 \\
\hline Bacillus spp. & 0 & 0 & 0 & 0 & 0 & 0 & 1 & 0.8 \\
\hline Culture negative & 65 & 40.1 & 59 & 36.9 & 28 & 21.4 & 29 & 24.0 \\
\hline Total & 162 & 100 & 160 & 100 & 131 & 100 & 121 & 100 \\
\hline
\end{tabular}

No = number; VG1 and N-VG1 = Vaccinate Group1 and Not-Vaccinated Group1, respectively; VG2 and N-VG2 = Vaccinate Group1 and Not-Vaccinated Group2, respectively. Herd A: received two doses of vaccine pre-calving; Herd B: received two doses of vaccine pre-calving and one post-calving

Table 2 Udder health status in primiparous Mediterranean buffalos within the two study herds (A and B) in association with Staphylococcus aureus detection in composite milk samples

\begin{tabular}{|c|c|c|c|c|c|c|c|c|c|}
\hline \multicolumn{10}{|l|}{ Herd A } \\
\hline \multirow[b]{2}{*}{ Udder health status } & \multirow[b]{2}{*}{ Groups } & \multicolumn{2}{|c|}{10 DIM } & \multicolumn{2}{|c|}{30 DIM } & \multicolumn{2}{|l|}{60 DIM } & \multicolumn{2}{|c|}{90 DIM } \\
\hline & & No. & Percent & No. & Percent & No. & Percent & No. & Percent \\
\hline \multirow[t]{2}{*}{$C M$} & VG1 & $0 / 30$ & 0 & $0 / 30$ & 0 & $2 / 30$ & 6.6 & $0 / 28$ & 0 \\
\hline & N-VG1 & $0 / 30$ & 0 & $2 / 30$ & 6.6 & $0 / 28$ & 0 & $0 / 28$ & 0 \\
\hline \multirow[t]{2}{*}{ SCM } & VG1 & $0 / 30$ & 0 & $0 / 30$ & 0 & $0 / 30$ & 0 & $4 / 28$ & 14.3 \\
\hline & N-VG1 & $0 / 30$ & 0 & $0 / 30$ & 0 & $0 / 28$ & 0 & $3 / 28$ & 10.7 \\
\hline \multirow[t]{2}{*}{$\mid \mathrm{Ml}$} & VG1 & $0 / 30$ & 0 & $5 / 30$ & 16.7 & $16 / 30$ & 53.3 & $22 / 28$ & 78.6 \\
\hline & N-VG1 & $1 / 30$ & 3.3 & $8 / 30$ & 26.6 & $15 / 28$ & 53.6 & $23 / 28$ & 82.1 \\
\hline \multirow[t]{2}{*}{ Healthy } & VG1 & $30 / 30$ & 100 & $25 / 30$ & 83.3 & $12 / 30$ & 40.0 & $2 / 28$ & 7.1 \\
\hline & N-VG1 & 29/30 & 96.7 & $20 / 30$ & 66.7 & $13 / 28$ & 46.4 & $2 / 28$ & 7.1 \\
\hline \multicolumn{10}{|l|}{ Herd B } \\
\hline \multirow[t]{2}{*}{$C M$} & VG2 & $0 / 30$ & 0 & $0 / 30$ & 0 & $0 / 28$ & 0 & $0 / 27$ & 0 \\
\hline & N-VG2 & $0 / 30$ & 0 & $0 / 27$ & 0 & $0 / 23$ & 0 & $0 / 20$ & 0 \\
\hline \multirow[t]{2}{*}{ SCM } & VG2 & $0 / 30$ & 0 & $2 / 30$ & 6.6 & $1 / 28$ & 3.6 & $3 / 27^{a}$ & 11.1 \\
\hline & N-VG2 & $3 / 30$ & 10.0 & $4 / 27$ & 14.8 & $3 / 23$ & 13.0 & $5 / 20^{b}$ & 25.0 \\
\hline \multirow[t]{2}{*}{ IMI } & VG2 & $2 / 30$ & 6.6 & $12 / 30$ & 40.0 & $14 / 28^{a}$ & 50.0 & $21 / 27$ & 77.8 \\
\hline & N-VG2 & $1 / 30$ & 3.3 & $15 / 27$ & 55.5 & $20 / 23^{b}$ & 86.9 & $13 / 20$ & 65.0 \\
\hline \multirow[t]{2}{*}{ Healthy } & VG2 & $28 / 30$ & 93.3 & $16 / 30$ & 53.3 & $13 / 28^{c}$ & 19.1 & $3 / 27$ & 11.1 \\
\hline & N-VG2 & $26 / 30$ & 86.7 & $8 / 27$ & 29.3 & $0 / 23^{d}$ & 0 & $2 / 20$ & 10.0 \\
\hline
\end{tabular}

VG1 and N-VG1 = Vaccinate Group1 and Not-Vaccinated Group1, respectively; VG2 and N-VG2 = Vaccinate Group1 and Not-Vaccinated Group2, respectively. CM = Clinical Mastitis; SCM = Subclinical Mastitis; IMI = Intramammary infection; DIM = Days in Milk. Herd A: received two doses of vaccine pre-calving; Herd B: received two doses of vaccine pre-calving and one post-calving. Animals affected by mastitis (SCM or CM) were considered in end- point. ${ }^{a, b} P<0.01$, ${ }^{c, d} P<0.001$ 
N-VG2 (16.7\%, 5/30). Regarding the prevalence of MB affected by IMI or with healthy udder, data are reported in detail in Table 2; no statistically significant difference between prevalence of IMI or healthy udder was found in VG1 and N-VG1 at any sampling time.

Details regarding of the effects on SCC and milk yield are reported in Table 3. Briefly, during the protocol A, the overall averages of $\log _{10}$ SSC values observed during the whole lactation were $4.93 \pm 4.71 \log _{10}$ cells $/ \mathrm{mL}$ in VG1 and $4.95 \pm 4.78 \log _{10}$ cells/mL in N-VG1 (IMI + healthy udder $\mathrm{MB}$ ); no statistically significant difference was found between the two values as well as between the daily mean values of SCC recorded at all sampling times in VG1 and N-VG1. Regarding the milk yields, the overall mean values observed in the same animals (IMI + healthy udder $\mathrm{MB}$ ) during the entire investigation were $9.70 \pm 2.07 \mathrm{~L} /$ day in VG1 and $9.35 \pm 1.57 \mathrm{~L} /$ day in $\mathrm{N}$ VG1; no statistically significant difference was recorded for this parameter at any sampling time.

\section{Protocol B}

The $45.8 \%(55 / 120)$ and $50.8 \%(61 / 120)$ of the samples cultured were positive for $S$. aureus in VG2 and N-VG2, respectively. The prevalence of positive-cultures for $S$. aureus in VG2 was 6.6\% (2/30) at 10 DIM and increased up to $46.7 \%(14 / 30), 53.6 \%(15 / 28)$ and $88.9 \%(24 / 27)$ at 30, 60 and 90 DIM, respectively; in N-VG2 it was instead of $13.3 \%$ (4/30), $70.4 \%$ (19/27), $100 \%(23 / 23)$ and $90.0 \%(18 / 20)$ at 10, 30, 60 and 90 DIM, respectively; a statistically significant difference was found between VG2 and N-VG2 at 60 DIM $(P<0.001)$.

Details regarding of the effects of $S$. aureus on udder health are reported in detail in Table 2. No CM due to $S$. aureus were recorded during the entire protocol in both groups (VG2 and N-VG2). SCM were detected at 30, $(6.6 \%, 2 / 30), 60(3.6 \%, 1 / 28)$ and 90 DIM $(11.1 \%, 3 / 27)$ in VG2, while in N-VG2, they were detected at each sampling time with a prevalence ranged between $10 \%$ and $25 \%$. A statistically significant difference was found at 90 DIM $(P<0.01)$. During the first 90 DIM, a statistically significant difference between the incidence of animals affected by mastitis in was also recorded (VG2: 20.0\% 6/30 and N-VG2: 50.0\% - 15/30, $P<0.05)$. According to the mixed logistic model the VG2 animals had less $23 \%$ of probability to develop mastitis compared to N-VG2 $(P<0.001)$. Regarding the prevalence of MB affected by IMI and with healthy udder a statistically significant difference between prevalence of $\mathrm{MB}$ affected by IMI in VG2 and N-VG2 was found at 60 DIM $(P<0.01)$, while between healthy udder at 60 DIM $(P<0.001)$.

Details regarding of the effects on SCC and milk yield are reported in Table 3. Briefly, during the protocol B, the overall average of $\log _{10}$ SSC values observed during the whole lactation were $4.84 \pm 4.60 \log _{10}$ cells $/ \mathrm{mL}$ in VG2 and $4.97 \pm 4.75 \log _{10}$ cells/mL in N-VG2 (IMI + healthy udder $\mathrm{MB}$ ); a statistically significant difference was found between the two values $(P<0.05)$. A statistically significant difference was also recorded between daily mean values of SCC in VG2 and N-VG2 at 10 $(P<0.05), \quad 30 \quad(P<0.05), 150 \quad(P<0.05)$ and 240 DIM $(P<0.01)$. Regarding the milk yields, the overall mean values observed in the same animals during the entire investigation were $10.37 \pm 2.18 \mathrm{~L} / \mathrm{d}$ in VG2 and $10.03 \pm$ $2.28 \mathrm{~L} / \mathrm{d}$ in N-VG2; no statistically significant difference was recorded at any sampling time between the two groups. The mixed linear model confirmed a significant

Table 3 Daily means somatic cell count values ( $\log _{10}$ cells/mL \pm SD) and milk yields (Litres \pm SD) detected in VGs and N-VGs in both the herds of primiparous Mediterranean buffalo during 240 days in milk of study

\begin{tabular}{|c|c|c|c|c|c|c|}
\hline \multirow[b]{2}{*}{ Item } & \multicolumn{6}{|l|}{ DIM } \\
\hline & 10 & 30 & 60 & 90 & 150 & 240 \\
\hline \multicolumn{7}{|c|}{ Herd A - $\log _{10}$ SCC } \\
\hline VG1 & $4.84( \pm 4.51)$ & $4.73( \pm 4.62)$ & $4.82( \pm 4.54)$ & $5.10( \pm 4.75)$ & $5.02( \pm 4.90)$ & $5.08( \pm 4.95)$ \\
\hline N-VG1 & $4.88( \pm 4.65)$ & $4.80( \pm 4.68)$ & $4.87( \pm 4.77)$ & $5.05( \pm 4.92)$ & $4.98( \pm 4.89)$ & $5.09( \pm 4.78)$ \\
\hline \multicolumn{7}{|c|}{ Herd B - $\log _{10}$ SCC } \\
\hline VG2 & $4.86( \pm 4.60)^{a}$ & $4.67( \pm 4.45)^{a}$ & $4.86( \pm 4.71)$ & $4.97( \pm 4.66)$ & $4.84( \pm 4.51)^{\mathrm{a}}$ & $4.85( \pm 4.67)^{c}$ \\
\hline N-VG2 & $4.99( \pm 4.74)^{b}$ & $4.94( \pm 5.01)^{b}$ & $4.87( \pm 4.69)$ & $4.87( \pm 4.73)$ & $4.98( \pm 4.67)^{\mathrm{b}}$ & $5.15( \pm 4.64)^{c}$ \\
\hline \multicolumn{7}{|c|}{ Herd A -Milk Yields } \\
\hline VG1 & $7.85( \pm 0.85)$ & $13.10( \pm 2.56)$ & $13.04( \pm 2.77)$ & $12.19( \pm 2.59)$ & $8.63( \pm 2.13)$ & $3.37( \pm 1.51)$ \\
\hline N-VG1 & $7.31( \pm 2.36)$ & $12.89( \pm 2.20)$ & $13.20( \pm 2.21)$ & $11.63( \pm 1.48)$ & $8.01( \pm 0.56)$ & $3.05( \pm 0.59)$ \\
\hline \multicolumn{7}{|c|}{ Herd B -Milk Yields } \\
\hline VG2 & $8.50( \pm 2.04)$ & $13.39( \pm 2.27)$ & $13.66( \pm 2.15)$ & $13.87( \pm 2.89)$ & $8.84( \pm 1.94)$ & $3.98( \pm 1.77)$ \\
\hline $\mathrm{N}-\mathrm{VG} 2$ & $7.7( \pm 2.12)$ & $13.08( \pm 2.53)$ & $13.10( \pm 2.83)$ & $12.41( \pm 2.34)$ & $9.57( \pm 4.89)$ & $4.34( \pm 1.57)$ \\
\hline
\end{tabular}

VG1 and N-VG1 = Vaccinate Group1 and Not-Vaccinated Group1, respectively; VG2 and N-VG2 = Vaccinate Group1 and Not-Vaccinated Group2, respectively; DIM = days in milk; ${ }^{a, b} P<0.05,{ }^{c, d} P<0.01$. Herd A: received two doses of vaccine pre-calving; Herd $B$ : received two doses of vaccine pre-calving and one post-calving 
difference between mean SCC of VG2 and N-VG2 at 150 (-66611.1, $P<0.001)$ and $240(-76444.4, P<0.01)$ DIM. No difference where instead detected regarding milk yields.

Finally, combining the two field trial, the mean difference of SCC between VG2 and N-VG2 was significantly higher than the analogous difference between VG1 and N-VG1 (-19778.06, $P<0.05)$. No significant differences were instead observed regarding the milk yields.

\section{Discussion}

For the purposes of the analysis outlined in this paper, the efficacy of two different regimens of vaccination, based on the use of a multivalent commercial inactivated bovine vaccine against $S$. aureus mastitis, was compared for the first time in $\mathrm{MB}$ during the lactation. Estimation of vaccine effects against contagious infections and mastitis under field conditions is an interesting challenge in species like $\mathrm{MB}$ where the knowledges regarding the preventions of mastitis are truly rare.

The high prevalence of mastitis due to $S$. aureus mono-infection reported in these ruminants [1], has been confirmed also in the present investigation. In this context, the influence of the dilution effects of $S$. aureus due to composite milk samples use has been not considered substantial, as described for cows [26]. Instead, the lack of quarter milk samples could represent a restriction of the present study because of lower sensibility of IMI detection and loss of useful information on quarter level (e.g. new infection rate, bacteriological cure rate, duration of the infections).

During the survey, only primiparous MB were enrolled because our previous studies showed a high-frequency isolation of $S$. aureus in first lactating animals between 30 and 90 DIM $[1,2,5]$ and also to exclude the potential negative influence of a poor management of dry period. The complete lack of previous knowledge concerning preventive vaccination against mastitis in $\mathrm{MB}$, lead the authors in a careful and methodical approach for the evaluation of the outcomes of a vaccine produced in origin for dairy cows; for this reason, two different regimens were carried out according to the manufacturer's instructions verifying the tolerance of the animals to the product, the effects on udder health, the milk quality and yields.

As described by Schukken et al. [13] for cows, also for $\mathrm{MB}$ the analysis of the data regarding the efficacy estimates was focused on the animals that received the full vaccination protocols; despite the ambition to vaccinate the animals according to the vaccination schemes planned, some difficulties were detected to achieve the necessary number of vaccine doses planned because of a continuous and precise scheduling required, inaccurate estimation of duration of pregnancy, early loss of pregnancy, abortions or $\mathrm{MB}$ calving pretermly. Given that incomplete vaccination due to the reasons described could be a common finding also in many $\mathrm{MB}$ herds, it is possible that our estimates of vaccine efficacy could be overestimated compared with the efficacy observed under true field conditions.

The importance of $S$. aureus as a contagious pathogen has been widely recognized both in dairy buffaloes and cows because cause of mastitis and relevant economic losses due to its negative influence on milk quality and yields $[2,6]$. A previous study investigating clinical relevance and genotype of $S$. aureus in $\mathrm{MB}$, elucidated that (1) genotype B was the only one detected, (2) it was associated to high within-herd prevalence (up to 55\%) and incidence (up to $20.4 \%$, during the whole lactation period), (3) it was responsible of contagious mastitis [2]. As well established also for cows, some strains of $S$. aureus (e.g. genotype B) are related to high contagiousness, causing herd problems with a within-herd prevalence of up to $87 \%$ [27]. A high prevalence and incidence of positive MB for $S$. aureus was also detected in both protocols and groups of the current investigation confirming the high-frequency isolation of this pathogen. Moreover, considering the overall cultured milk samples, S. aureus was also the most frequently isolated bacterium in both the herds and groups followed by coagulase-negative staphylococci. The result confirms a similar findings recently observed both in MB [2] and cows herds [28] where the percentage of isolation of coagulase-negative staphylococci and Streptococci were indeed considerably lower in herds positive for S. aureus showing high contagiousness and pathogenicity.

A significant difference in the overall rate of mastitis, comparing unvaccinated and vaccinated $\mathrm{MB}$, was observed only in herd B. The administrations of 3 doses of vaccine, may significantly reduce the incidence of mastitis within the herd; the difference seems to be related to the lower prevalence of SCM detected at any sampling time in the VG2 than N-VG2 and achieving a significant peak at 90 DIM. Therefore, the novel contribution of the current study has been to show encouraging results regarding the efficacy of the inactivate vaccine on $\mathrm{MB}$, in contrast with some previous similar studies on staphylococcal vaccination in dairy cows described relatively low vaccine efficacy or no vaccine efficacy at all [15, 17]. However, it is necessary to underline that another of the restrictions of the present investigation may be represented by the chosen study design: indeed only the direct vaccine effects, without attempt to estimate the overall population estimates, were described; as a consequence, it possible that our outcomes may be overestimated if compared with the truly observed vaccine efficacy under overall population conditions. Furthermore, the presence of $\mathrm{CM}$ in herd A (VG and CG) suggests that the protocol 
based on 2 vaccine administrations had not effect to prevent the appearance of clinical signs; on the contrary, in herd $\mathrm{B}$, no difference has been found in both the groups considered overtime and therefore it has been not possible to evaluate this effect. The finding merits further scientific attention, because of the small number of animals enrolled and the lack of previous studies performed on MB useful to compare the results observed.

Regarding IMI due to $S$. aureus, a high prevalence of MB infected was recorded in both the protocols although lower rate of infection was observed in VGs than N-VGs in almost all the samplings. During the investigation a higher prevalence of infections was always observed between 60 and 90 DIM in both the protocols and groups; these effects may have been caused by mixing primiparous with pluriparous animals in the herds, as initiated by the farmer between 40 and 50 DIM. It is interesting to highlight how the administration of the third dose seems to decrease significantly the prevalence of IMI between VG2 and $\mathrm{N}-\mathrm{VG} 2$ at 60 DIM, conversely to the use of two doses. Moreover, the moderate reduction of IMI observed in this sampling time, may have consequently reduced the mastitis prevalence observed in the successive control time; the finding described may be also supported by the significant higher percentages of healthy udder $\mathrm{MB}$ recorded in VG2 than N-VG2 at 60 DIM. The results observed seem less encouraging than those recently described for cows where vaccination resulted in moderate reduction in incidence of new infections and more pronounced reduction in duration although in presence of an efficacy for transmission still relatively low [13]. As reported by several studies in cows [27-29], also in our investigation the vaccine effects on IMI may be influenced by farm-specific characteristics, such as strain types and farm management practices, and not be only ascribed to the vaccine's properties. Excellent preventive herd management strategies may contribute to significantly reduce the overall herd exposure to $S$. aureus both in $\mathrm{MB}$ and cows $[2,13,30]$, resulting in an important contribution to indirect vaccine efficacy.

Regarding the effects of vaccination on milk yield and quality, no significant differences were observed between the overall mean milk yields and daily mean production between the two groups of both protocols, while a significant difference between the two regimens was observed regarding SCC. As reported in Table 3, the protocol A was not able to positively influence SCC values in VG1 compared with N-VG1, differently from the protocol $\mathrm{B}$, in which the additional vaccine's dose may have produced an additional immunization of VG able to reduce $S$. aureus multiplication in the mammary gland as reported also for cows [15]; therefore, the additional administration seems to significantly decrease SCC values in the successive days in milk and take part in the significant difference observed on overall SCC mean values. Moreover, the decrease of $S$. aureus' negative effects seems to be also confirmed by the lower prevalence of mastitis recorded from 30 to 90 DIM in VG2 than N-VG2.

\section{Conclusions}

The current study represents the first investigation evaluating direct effects of vaccination against $S$. aureus in dairy Mediterranean Buffalo. Even though this study included only a small number of animals, the use of inactivated vaccine by means of the protocol B (based on label use) shows encouraging results because associated with significant lower prevalence and incidence of mastitis as well as lower SCC values, than the protocol A (based on two doses). Nevertheless, the lack of largescale effects against IMI highlights that vaccination in $\mathrm{MB}$, as in cow, may be considered only an additional tool in the control of $S$. aureus infections on farms and that its use should be always associated with excellent farm management practices to successfully improve the infections control within the herd. The assessment of its effects on the entire dairy MB herd under field conditions, as well as the in-depth analysis of the infectious dynamics, warrant further scientific attention with the goal to fully understand the potentialities of this inactivated vaccine in dairy buffalo management.

\section{Abbreviations \\ BMC: Bacteriological milk culture; Cell: Cellules; CM: Clinical mastitis; CMT: California mastitis test; DIM: Days in milk; E. coli: Escherichia coli; h: Hour; IMI: Intramammary infection; MB: Mediterranean buffaloes; $\mathrm{mL}$ : Milliliter; N- VG: Not-vaccinated group; SAAC: Slime-associated antigenic complex; SCC: Somatic cell count; SCM: Subclinical mastitis; S. aureus: Staphylococcus aureus; VG: Vaccinated group}

\section{Acknowledgements}

The Authors acknowledge the precious collaboration of farmer during both the clinical trials.

\section{Funding}

The study was financially supported by Hipra (Rovato, Italy). The funding company participated in the conceptual aspect and design of the study and reviewing of the final version of the manuscript.

\section{Availability of data and materials}

All the data supporting the findings of the present study are included within the manuscript except for those regarding the California Mastitis Test routinely performed from each composite milk sample. The letters are available contacting the Corresponding Author on reasonable request.

\section{Authors' contributions}

$J G, A P, M P, G T \& P C$ participated in the conceptual aspect and design of the study. JG, MP, LDA, ADR \& PC involved in samples and data collection. JG, AP \& CS performed the samples analysis. JG, AP \& PC statistical evaluation and data interpretation. All authors provided consultation and coordination. JG, AP \& PC wrote the first draft of the manuscript, with all authors involved in reviewing. All authors read and approved the final version of the manuscript. 


\section{Competing interests}

The authors declare that they have no competing interests. The author affiliated with the private company did not influence in anyway the outcomes of the study. He was only involved in conceptual aspect and design of the study and reviewing of the final version of the manuscript. The company that he represents adheres to the Good Publication Practice guidelines for pharmaceutical companies (GPP3) ensuring that the publication is produced in a responsible and ethical manner.

\section{Consent for publication}

Not applicable.

\section{Ethics approval and consent to participate}

The study received the institutional approval by the Ethical Animal Care and Use Committee of University of Studies of Naples "Federico II" (n. 2016/ 0052967), moreover all the farmers involved was previously informed and in agreement about purposes and methods of the present investigation.

\section{Author details}

${ }^{1}$ Department of Veterinary Medicine and Animal Productions, University of Napoli "Federico II", Via Delpino 1, 80137 Naples, Italy. ${ }^{2}$ Istituto Zooprofilattico del Mezzogiorno, Via A. Jervolino 19, 81100, Tuoro, Caserta District, Italy. ${ }^{3}$ Veterinary practitioner, Caserta District 81100 , Italy. ${ }^{4}$ Hipra Italia s.r.l., Via Franciacorta 74, 25038 Rovato, Italy.

\section{Received: 3 August 2016 Accepted: 9 January 2017}

\section{Published online: 19 January 2017}

\section{References}

1. Guccione J, Cosandey A, Pesce A, Di Loria A, Pascale M, Piantedosi D, et al. Clinical outcomes and molecular genotyping of Staphylococcus aureus isolated from milk samples of dairy primiparous Mediterranean buffaloes (Bubalus bubalis). J Dairy Sci. 2014;97:7606-13.

2. Guccione J, Pesce A, Pascale M, Tommasini N, Garofalo F, Di Loria A, et al. Short communication: Effects of systemic treatment with penethamate hydriodide on udder health and milk yields in dry primiparous Mediterranean buffaloes (Bubalus bubalis). J Dairy Sci. 2014;97:2219-25.

3. Moroni P, Rossi CS, Pisoni G, Bronzo V, Castiglioni B, Boettcher PJ. Relationships between Somatic Cell Count and Intramammary Infection in Buffaloes. J Dairy Sci. 2006;89:998-1003.

4. Fagiolo A, Lai O. Mastitis in buffalo. Ital J Anim Sci. 2007;6:200-6.

5. Guccione J, Perreten V, Steiner A, Thomann A, Pesce A, Ciaramella P, et al. Short communication: Role of Streptococcus pluranimalium in Mediterranean buffaloes (Bubalus bubalis) with different udder health statuses. J Dairy Sci. 2016;99:2945-9.

6. Sears PM, McCarthy KK. Management and treatment of staphylococcal mastitis. Vet Clin North Am Food Anim Pract. 2003:19:171-85.

7. Barkema HW, Schukken YH, Zadoks RH. Invited review: The role of cow, pathogen, and treatment regimen in the therapeutic success of bovine Staphylococcus aureus mastitis. J Dairy Sci. 2006;89:1877-95.

8. Barkema HW, Green MJ, Bradley AJ, Zadoks RN. Invited review: The role of contagious disease in udder health. J Dairy Sci. 2009;92:4717-29.

9. Keefe G. Update on control of Staphylococcus aureus and Streptococcus agalactiae for management of mastitis. Vet Clin North Am Food Anim Pract. 2012;28:203-16.

10. Pérez MM, Prenafeta A, Valle J, Penadés J, Rota C, Solano C, et al. Protection from Staphylococcus aureus mastitis associated with poly- $\mathrm{N}$-acetyl beta-1,6 glucosamine specific antibody production using biofilm-embedded bacteria. Vaccine. 2009;27:2379-86.

11. Pereira UP, Oliveira DG, Mesquita LR, Costa GM, Pereira LJ. Efficacy of Staphylococcus aureus vaccines for bovine mastitis: A systematic review. Vet Microbiol. 2011;148:117-24.

12. Daum RS, Spellberg B. Progress toward a Staphylococcus aureus vaccine. Clin Infect Dis. 2012;54:560-7.

13. Schukken YH, Bronzo V, Locatelli C, Pollera C, Rota N, Casula A, et al. 2014 Efficacy of vaccination on Staphylococcus aureus and coagulase-negative staphylococci intramammary infection dynamics in 2 dairy herds. J Dairy Sci. 2014;97:5250-64

14. Bradley AJ, Breen JE, Payne B, White V, Green MJ. An investigation of the efficacy of a polyvalent mastitis vaccine using different vaccination regimens under field conditions in the United Kingdom. J Dairy Sci. 2015;98: 1706-20.

15. Middleton JR, Luby CD, Adams DS. Efficacy of vaccination against staphylococcal mastitis: A review and new data. Vet Microbiol. 2009:134:192-8.

16. Freick M, Frank Y, Steinert K, Hamedy A, Passarge O, Sobiraj A. Mastitis vaccination using a commercial polyvalent vaccine or a herd-specific Staphylococcus aureus vaccine. Results of a controlled field trial on a dairy farm. Tierarztl Prax Ausg G Grosstiere Nutztiere. 2016;44:219-29.

17. Landin H, Mörk MJ, Larsson M, Waller KP. Vaccination against Staphylococcus aureus mastitis in two Swedish dairy herds. Acta Vet Scand. 2015:57:81.

18. Syring C, Boss R, Reist M, Bodmer M, Hummerjohann J, Gehrig P, et al. Bovine mastitis: The diagnostic properties of a PCR-based assay to monitor the Staphylococcus aureus genotype B status of a herd, using bulk tank milk. J Dairy Sci. 2012;95:3674-82

19. Thrusfield M. Surveys. In: Thrusfield M, editor. Veterinary epidemiology. London: UK: Blackwell science Ltd; 2007. p. 228-42.

20. Ciaramella P, Guccione J. Esame del buffalo di razza mediterranea italiana. In: Ciaramella P, editor. Semeiologia Clinica Veterinaria. Milano, Italy.: Poletto Editore srl; 2014. p. 524-32.

21. European Medicine Agency ${ }^{\oplus}$, Science medicine health. London 2012. http:// www.ema.europa.eu/ema/index.jsp?curl=pages/medicines/veterinary/ medicines/000130/vet_med_000187.jsp\&mid=WC0b01ac058008d7a8. Accessed 25 Sept 2016.

22. National Mastitis Council. Procedures for Collecting Milk Samples in Microbiological Procedures for the Diagnosis of Bovine Udder Infection and Determination of Milk Quality. Madison, WI: National Mastitis Council Inc: 2004

23. Tripaldi C, Paolacci G, Mairelli M, Catta M, Orlandini S, Amatiste S, et al. Effects of mastitis on buffalo milk quality. Asian-australas J Anim Sci. 2010; 23:1319-24.

24. Fossomatic ${ }^{T M}$ FC. Somatic cell count. Hilleroed Denmark 2012 http://www. foss.dk/industry-solution/central-milk-testing/brochures-and-data-sheets. Accessed 01 October 2012

25. National Mastitis Council. Laboratory Handbook on Bovine Mastitis. Rev. ed. National Mastitis Council Inc., Madison, Wl. 1999.

26. Lam TJ, Van Vliet JH, Schukken YH, Grommers FJ, Van Velden-Russcher A, Barkema HW, et al. The effect of discontinuation of postmilking teat disinfection in low somatic cell count herds. II. Dynamics of intramammary infections. Vet Q. 1997;19:47-53.

27. Michel A, Syring C, Steiner A, Graber HU. Intramammary infections with the contagious Staphylococcus aureus genotype B in Swiss dairy cows are associated with low prevalence of coagulase-negative staphylococci and Streptococcus spp. Vet J. 2011;188:313-7.

28. Lam TJGM, van Wuijckhuise LA, Franken P, Morselt ML, Hartman EG, Schukken $\mathrm{YH}$. Use of composite milk samples for diagnosis of Staphylococcus aureus mastitis in dairy cattle. J Am Vet Med Assoc. 1996; 208:1705-8.

29. Barlow JW, Zadoks RN, Schukken YH. Effect of lactation therapy on Staphylococcus aureus transmission dynamics in two commercial dairy herds. BMC Vet Res. 2013;9:28-40

30. Guccione J, Carcasole C, Alsaaod M, D'Andrea L, Di Loria A, De Rosa A, Ciaramella P, Steiner A. Assessment of foot health and animal welfare: clinical findings in 229 dairy Mediterranean Buffaloes (Bubalus bubalis) affected by foot disorders. BMC Vet Res. 2016;12:(1).

\section{Submit your next manuscript to BioMed Central and we will help you at every step:}

- We accept pre-submission inquiries

- Our selector tool helps you to find the most relevant journal

- We provide round the clock customer support

- Convenient online submission

- Thorough peer review

- Inclusion in PubMed and all major indexing services

- Maximum visibility for your research

Submit your manuscript at www.biomedcentral.com/submit
) Biomed Central 